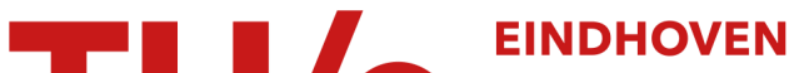 UNIVERSITY OF TECHNOLOGY
}

\section{On the impact of optimal modulation and FEC overhead on future optical networks}

\section{Citation for published version (APA):}

Alvarado, A., Ives, D. J., Savory, S. J., \& Bayvel, P. (2016). On the impact of optimal modulation and FEC overhead on future optical networks. Journal of Lightwave Technology, 34(9), 2339-2352.

https://doi.org/10.1109/JLT.2016.2517699

DOI:

10.1109/JLT.2016.2517699

Document status and date:

Published: 01/05/2016

\section{Document Version:}

Publisher's PDF, also known as Version of Record (includes final page, issue and volume numbers)

\section{Please check the document version of this publication:}

- A submitted manuscript is the version of the article upon submission and before peer-review. There can be important differences between the submitted version and the official published version of record. People interested in the research are advised to contact the author for the final version of the publication, or visit the $\mathrm{DOI}$ to the publisher's website.

- The final author version and the galley proof are versions of the publication after peer review.

- The final published version features the final layout of the paper including the volume, issue and page numbers.

Link to publication

\section{General rights}

Copyright and moral rights for the publications made accessible in the public portal are retained by the authors and/or other copyright owners and it is a condition of accessing publications that users recognise and abide by the legal requirements associated with these rights.

- Users may download and print one copy of any publication from the public portal for the purpose of private study or research.

- You may not further distribute the material or use it for any profit-making activity or commercial gain

- You may freely distribute the URL identifying the publication in the public portal.

If the publication is distributed under the terms of Article 25fa of the Dutch Copyright Act, indicated by the "Taverne" license above, please follow below link for the End User Agreement:

www.tue.nl/taverne

Take down policy

If you believe that this document breaches copyright please contact us at:

openaccess@tue.nl

providing details and we will investigate your claim. 


\title{
On the Impact of Optimal Modulation and FEC Overhead on Future Optical Networks
}

\author{
Alex Alvarado, Senior Member, IEEE, David J. Ives, Member, OSA, \\ Seb J. Savory, Senior Member, IEEE, Member, OSA, and Polina Bayvel, Senior Member, IEEE, Senior Member, OSA
}

\begin{abstract}
The potential of optimum selection of modulation and forward error correction (FEC) overhead $(\mathrm{OH})$ in future wavelength-routed nonlinear optical mesh networks is studied from an information theory perspective. Different network topologies are studied as well as both ideal soft-decision (SD) and hard-decision (HD) FEC based on demap-and-decode (bit wise) receivers. When compared to the somewhat standard assumption of QPSK with 7\% $\mathrm{OH}$, the results show large gains in network throughput. When compared to SD-FEC, HD-FEC is shown to cause network throughput losses of $12 \%, 15 \%$, and $20 \%$ for a national, continental, and transcontinental topology, respectively. Furthermore, it is shown that for national and continental network topologies, using one modulation format and only two $\mathrm{OHs}$ achieves at least $75 \%$ of the maximum theoretical throughput. This is in contrast with the infinite number of $\mathrm{OHs}$ required in the ideal case. The obtained optimal OHs are between $5 \%$ and $80 \%$, highlighting the advantage of using FEC with high OHs.
\end{abstract}

Index Terms-Bit-wise receivers, channel coding, forward error correction, modulation, optical networks, soft-decision.

\section{INTRODUCTION AND MOTIVATION}

$\mathbf{T}$ he rapid rise in the use of the Internet has led to increasing traffic demands putting severe pressure on backbone networks. The transport backbone of the Internet is made of optical mesh networks, where optical fiber links connect nodes formed of reconfigurable optical add drop multiplexers (ROADMs). Studying the ultimate transmission limits of optical mesh networks as well as the optimal utilization of the installed network resources is key to avoid the so-called "capacity crunch" [1], [2].

Installed optical mesh networks use wavelength routing to transparently connect source and destination transceivers. The quality of the optical communication signal degrades due to transmission impairments, which in turn limits the maximum

Manuscript received October 21, 2015; revised December 10, 2015; accepted January 8, 2016. Date of publication January 12, 2016; date of current version March 18, 2016. This work was supported by the Engineering and Physical Sciences Research Council through the project INSIGHT EP/L026155/1 and the Program Grant UNLOC EP/J017582/1. This paper was presented in part at the Optical Fiber Communication Conference, Los Angeles, CA, USA, March 2015.

A. Alvarado, D. J. Ives, and P. Bayvel are with the Optical Networks Group, Department of Electronic and Electrical Engineering, University College London, London WC1E 7JE, U.K. (e-mail: alex.alvarado@ieee.org; d.ives@ee.ucl.ac.uk; p.bayvel@ucl.ac.uk).

S. J. Savory was with the Optical Networks Group, Department of Electronic and Electrical Engineering, University College London, London WC1E 7JE, U.K. He is now with the Department of Engineering, Electrical Engineering Division, University of Cambridge, Cambridge CB3 OFA, U.K. (e-mail: sjs1001@cam.ac.uk).

Color versions of one or more of the figures in this paper are available online at http://ieeexplore.ieee.org.

Digital Object Identifier 10.1109/JLT.2016.2517699 achievable data rate. This degradation is usually due to the accumulated noise introduced by amplifiers in the link as well as nonlinear distortion due to neighboring WDM channels. Furthermore, the optical signals within a transparent wavelength routed network travel a variety of distances, and thus, experience different levels of signal degradation. The most conservative design strategy for an optical network is to choose the transceiver to operate error-free on the worst light path, i.e., for transmission between the furthest spaced nodes [3, p. 138], [4, Sec. 1], [5, Sec. 1]. Under this design paradigm, any route reconfiguration can be accommodated through the network. However, this leads to over provisioning of resources.

The increase in traffic demand together with the development of software-defined transceivers that can adapt the transmission parameters to the physical channel have increased the interest in designing networks that utilize the resources more efficiently. The degrees of freedom in the transceiver design include, e.g., the forward error correction (FEC) scheme, FEC overhead $(\mathrm{OH})$, modulation format, frequency separation (in flex-grid networks), launch power, and symbol rate (see [6, Fig. 3]). The network resources can be better utilized if these degrees of freedom are jointly optimized in conjunction with the routing of the optical light path through the network.

To cope with increasing capacity demands, future optical networks will use multilevel modulations and FEC. This combination is known as coded modulation (CM) and its design requires the joint optimization of the FEC and modulation format (see Fig. 1). The optimum receiver structure for $\mathrm{CM}$ is the maximum likelihood (ML) receiver, which finds the most likely coded sequence [7, Sec. 3.1]. The ML solution is in general impractical, and thus, very often the receiver is implemented as a (suboptimal) bit-wise (BW) receiver instead [7, Sec. 3.2]. In a BW receiver, hard or soft information on the code bits is calculated first, and then, an FEC decoder is used (see the receiver side of Fig. 1). In other words, practical receivers decouple the detection process: symbols are first converted into bits, and then, FEC decoding is applied. In this paper we consider BW receivers with both soft-decision FEC (SD-FEC) and hard-decision FEC (HD-FEC). BW receivers have been studied for optical communications in, e.g., [8]-[10]

Traditional analyses of optical networks assume a target preFEC bit error rate (BER), and thus, an HD-FEC with fixed $\mathrm{OH}$ is implicitly assumed. ${ }^{1}$ Although the most common value for the $\mathrm{OH}$ is $7 \%$, higher $\mathrm{OHs}$ have become increasingly popular. Furthermore, the use of SD-FEC with high OHs (typically

\footnotetext{
${ }^{1}$ When SD-FEC is considered, however, pre-FEC BER does not determine the FEC OH, as recently shown in [11].
} 


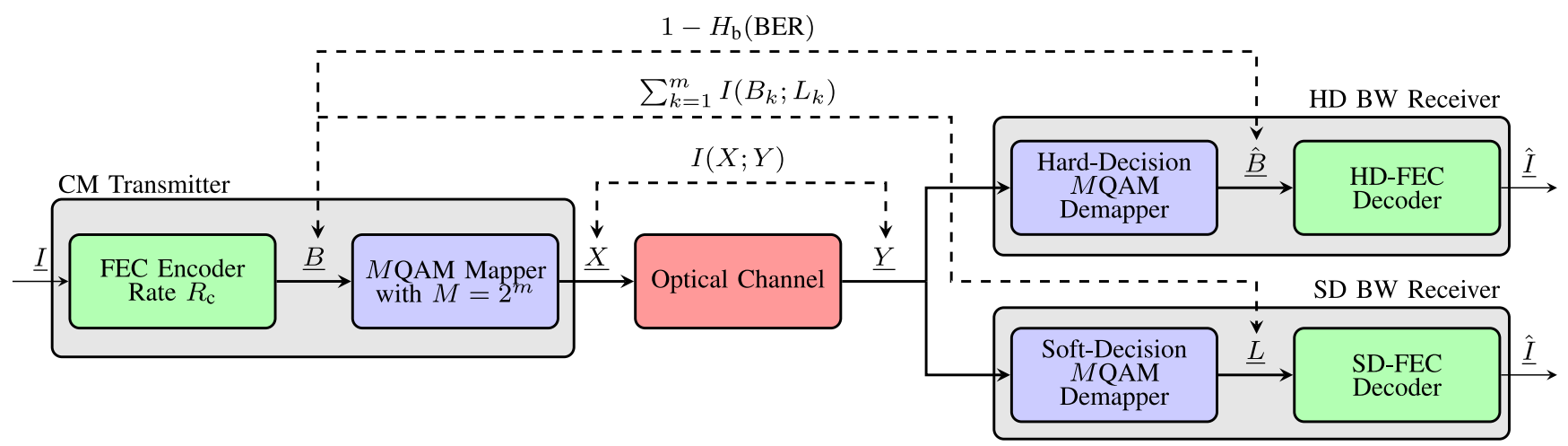

Fig. 1. CM system under consideration. At the transmitter, a binary FEC code is concatenated with a $M$-ary QAM modulator. After transmission, the noisy received symbols are demapped and then decoded by a BW receiver. When the demapper makes HDs on the symbols, an HD-FEC is used. When the demapper computes log-likelihood ratios (SDs), an SD-FEC is assumed. The achievable rates discussed in Section IV are also shown.

around 20\%) is considered the most promising FEC alternative for $100 \mathrm{G}$ and $400 \mathrm{G}$ transceivers. Although from a theoretical point of view, fixing the $\mathrm{OH}$ is an artificial constraint that reduces flexibility of the CM design and reduces the network throughput, there are good reasons for fixing the $\mathrm{OH}$. The client rates are usually quantized to $10,40,100,400 \mathrm{~Gb} / \mathrm{s}$ for compatibility with the Ethernet standards. The symbol rate is also often fixed to accommodate the transmitted bandwidth into a given fixed-grid, leading to fixed OHs. Under these constraints, no full flexibility on the selection of OHs is possible. In this paper, however, we ignore these constraints and focus on finding the theoretically maximum network throughput.

To increase the network throughput, different approaches have been investigated in the literature. For example, [12] considered mixed line rates $(10,40,100 \mathrm{~Gb} / \mathrm{s})$ for the NSF mesh topology, [13] considered variable FEC OHs with fixed symbol rate and modulation formats, and [14] considered variable modulation format and SD-FEC OHs. Adaptive FEC based on practical codes was recently considered in [4] and [5]. Variable OHs with 16QAM and 64QAM were studied in [15], where probabilistically-shaped constellations were considered. The optimal modulation format based on an approximation for the maximum achievable rates of HD-FEC was considered in [16]. Adaptive FEC OH for time frequency packing transmission was studied in [17]-[20]. The problem of routing and spectrum assignment for flex-grid optical networks with orthogonal frequency-division multiplexing was studied in [21]. The key enabling technology for these approaches are software-defined transceivers, allowing for example to vary the modulation format and symbol rate, as done in [22]-[26]. In [20] and [27], a software defined transceiver with variable FEC was experimentally demonstrated.

To optimize the network design, a physical layer model is required. While in the past very simple models (e.g., reachbased models) were considered, recently, nonlinear effects have been taken into account using the Gaussian noise (GN) model [28]-[34]. In [35], the closed form solution of the GN model of [34] was used to adapt the routing and wavelength assignment problem, for a target pre-FEC BER, and four different modulation formats. The same GN model was used in [36] and [37] to jointly optimize power, modulation format, and carrier frequencies (flex-grid) for a fixed $\mathrm{OH}$. Numerical integration of the GN model was used in [38] to assess signal-to-noise ratio (SNR) and throughput optimization via power and modulation adaptation. The numerically integrated GN model in [38] was also used in [39] and [40] to sequentially optimize modulation format and power for a fixed FEC OH. The potential gains of adaptive FEC OH and modulation, or adaptive launch power and modulation were studied in [41].

In this paper, we study the problem of finding the optimal modulation and FEC OH from an information theory viewpoint. In particular, we use information theoretic quantities (i.e., achievable rates) and a realistic model for the nonlinear interference to study the maximum network throughput of optical mesh networks. For point-to-point links, and under a Gaussian assumption on the channel, the solution depends only on the end-to-end link SNR. For an optical network, however, the solution depends on the SNR distribution of the connections. Therefore, the theoretically optimum CM design is obtained when the modulation size and FEC OH are jointly designed across the network. Significant increases in network throughput are shown. Furthermore, practically relevant schemes (based on either one or two $\mathrm{OHs}$ ) are also considered, and their gap to the theoretical maximum is quantified. This paper extends our results in [42] by considering multiple network topologies as well as both HD- and SD-FEC.

This paper is organized as follows. In Section II the system model, network topologies, and physical layer model are described. In Section IV the optimal selection of modulation and coding is reviewed and the maximum network throughput is analyzed. In Section V practically relevant schemes are considered. Conclusion is drawn in Section VI.

\section{PRELIMINARIES}

\section{A. System Model}

We consider the CM transmitter shown in Fig. 1, where a binary FEC code maps the information bits $\underline{I}=\left[I_{1}, I_{2}, \ldots, I_{k_{c}}\right]$ into a sequence of code bits $\underline{B}=\left[B_{1}, B_{2}, \ldots, B_{n_{c}}\right]$, where $R_{c}=k_{c} / n_{c}$ is the code rate. At each discrete-time instant, $m$ code bits are mapped to a constellation symbols from a discrete constellation with $M=2^{m}$ constellation points. We 
consider polarization-multiplexed square QAM constellations with $m=2,4,6,8,10$ (i.e., $M$ QAM with $M \leq 1024$ ) in each polarization. The constellations are labeled by the binaryreflected Gray code. For an FEC encoder with code rate $R_{c}$ and a modulation format with $M$ symbols, the spectral efficiency (SE) per two polarizations is

$$
\mathrm{SE}=2 R_{c} m\left[\frac{\mathrm{bit}}{\text { symbol }}\right] .
$$

The FEC OH is

$$
\mathrm{OH}=100\left(\frac{1}{R_{c}}-1\right) \% .
$$

At the receiver side a $\mathrm{BW}$ receiver is used. ${ }^{2}$ In such a receiver, the noisy symbols are first demapped, and then, FEC decoding is performed. The FEC decoder gives an estimate of the transmitted bits $\hat{I}$ (see Fig. 1). Due to the separation of the detection process, BW receivers are suboptimal. They are, however, very common in practice due to the use of off-the-shelf FEC decoders.

In this paper we consider two types of FEC decoders: HDand SD-FEC. This naturally leads to two different BW receiver structures, shown on the right-hand side of Fig. 1. In an HD BW receiver, the demapper makes HDs on the bits (by making HDs on the symbols), which are then passed to an HD-FEC decoder. We assume that there is a random bit-level interleaver between the encoder and mapper, which we consider part of the FEC encoder (decoder).

In an SD BW receiver, the demapper calculates soft information on the code bits (also known as "soft bits"), which are then passed to an SD-FEC decoder. The soft information is usually represented in the form of logarithmic likelihood ratios (LLRs), defined as

$$
L_{q} \triangleq \log \frac{f_{Y \mid B_{q}}(y \mid 1)}{f_{Y \mid B_{q}}(y \mid 0)}, \quad q=1, \ldots, m,
$$

where $B_{q}$ is the $q$ th bit at the input of the mapper, and $f_{Y \mid B_{q}}(y \mid b)$, $b \in\{0,1\}$ is the channel transition probability. The sign of the LLRs corresponds to an HD on the bits, while its amplitude represents the reliability of the available information.

\section{B. Network Topologies}

In this paper we consider three networks shown in Figs. 2-4. The first one is the network topology for Deutsche Telekom Germany (DTG) [43, Sec. II], where the two core nodes per city (see [43, Fig. 1]) were merged into one. The second one is the reference 14-node, 21-link NSF mesh topology [39, Fig. 1], [38, Fig. 7]. The last topology is the Google B4 (GB4) network connecting data centers in [44, Fig. 1]. We chose to study these three topologies because they are representative of networks at three different scales: national, continental, and transcontinental.

\section{Physical Layer Model}

For the analysis in this paper, it is assumed that each node in the networks described in Section II-B is equipped with

\footnotetext{
${ }^{2}$ Also known as bit-interleaved CM receiver [7]
}

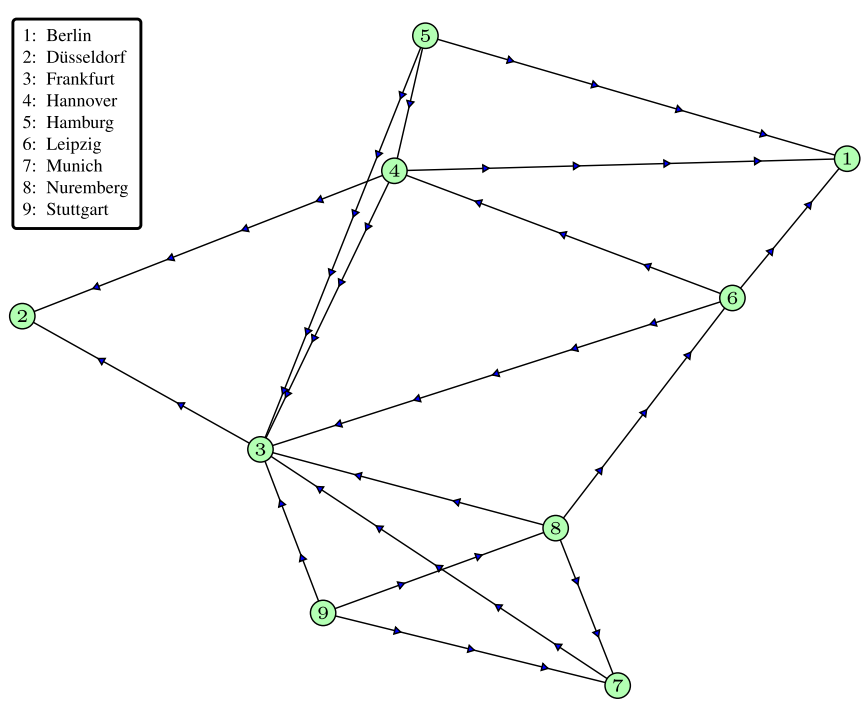

Fig. 2. DTG network formed by nine nodes representative of a national topology. The location of the amplifiers (in one direction) is shown as triangles.

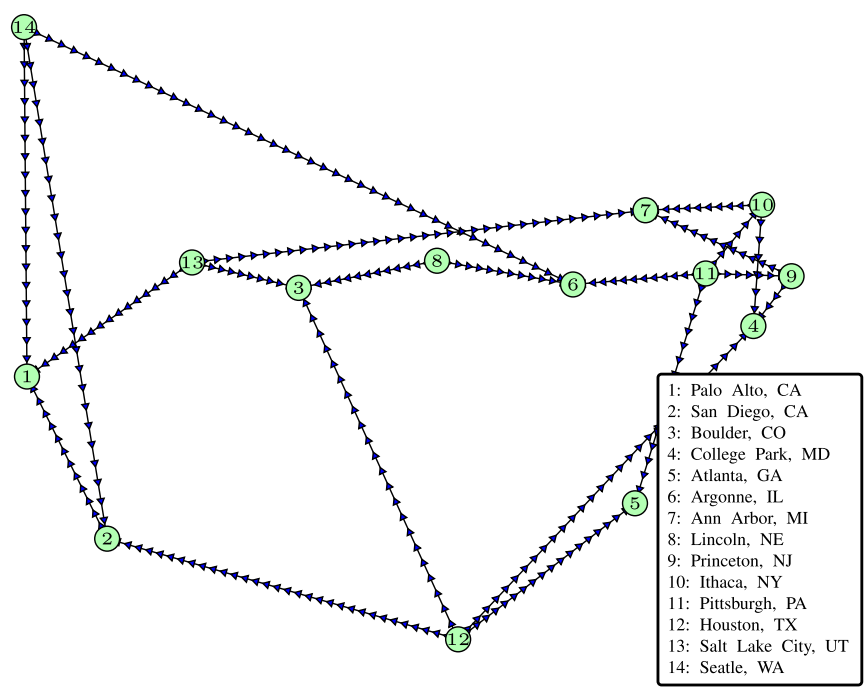

Fig. 3. NSF network formed by 14 nodes representative of a continental topology. The location of the amplifiers (in one direction) is shown as triangles.

multiple transceivers. Furthermore, it is also assumed that these transceivers are based on the structure in Fig. 1 and that they can ideally adapt the FEC OH and modulation order. We consider a fixed grid of $80 \mathrm{WDM}$ channels of $50 \mathrm{GHz}$ per fiber, which accounts for approximately the entire C-band. The symbol rate considered is 32 GBaud.

The nodes are connected with fiber pairs with standard singlemode fiber with parameters shown in Table I. Erbium-doped fiber amplifiers (EDFA) are regularly placed between the links, as shown in Figs. 2 and 3. ${ }^{3}$ The span length is $80 \mathrm{~km}$ and the EDFA noise figure is assumed to be $5 \mathrm{~dB}$.

Every link in the network is modeled using a channel model (see Fig. 1) that encompasses all the transmitter digital signal

\footnotetext{
${ }^{3}$ The amplifiers are not shown in Fig. 4 so as not to overcrowd the figure.
} 


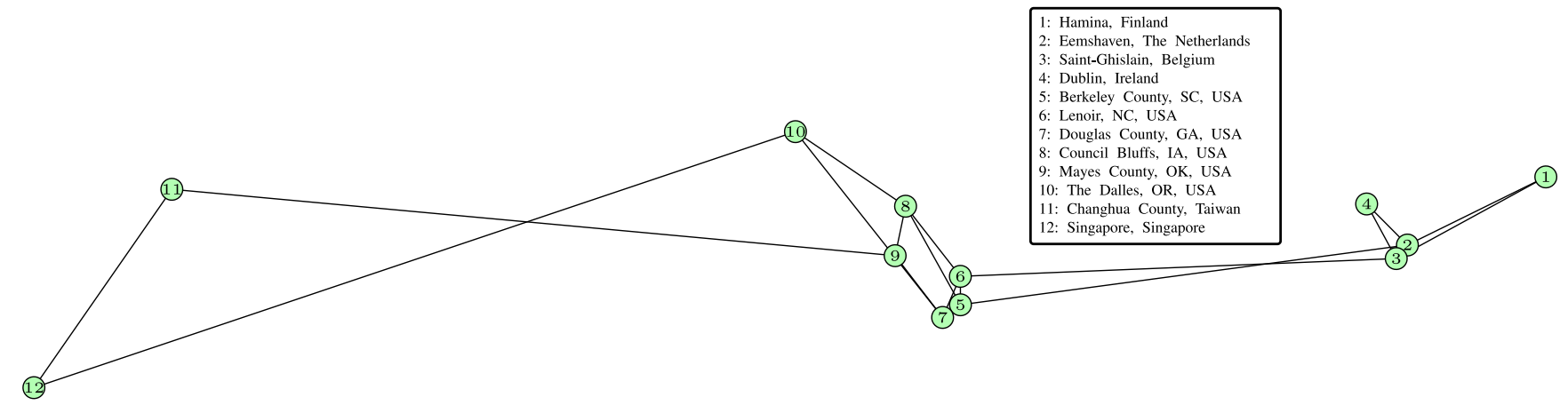

Fig. 4. GB4 network (amplifiers are not shown) formed by 12 nodes representative of a transcontinental topology.

TABLE I

SUMMARY OF SYSTEM PARAMETERS

\begin{tabular}{ll}
\hline \hline Parameter & Value \\
\hline Fiber attenuation & $0.22 \mathrm{~dB} / \mathrm{km}^{-1} \mathrm{~km}^{-1}$ \\
Dispersion parameter & $16.7 \mathrm{ps} \mathrm{nm}^{-1} \mathrm{~km}^{-1}$ \\
Fiber nonlinear coefficient & $1.3 \mathrm{~W}^{-1}$ \\
Span length & $80 \mathrm{~km}$ \\
EDFA noise figure & $5 \mathrm{~dB}$ \\
ASE Power per span $P_{\mathrm{ASE}}$ & $0.747 \mu \mathrm{W}$ \\
\hline Symbol rate & $32 \mathrm{Gbaud}$ \\
WDM channels & 80 \\
Channel separation & $50 \mathrm{GHz}$ \\
Pulse shape & $\mathrm{Nyquist}$ sinc pulses \\
SPM & Ideally compensated \\
ROADM Loss & $0 \mathrm{~dB}$ \\
\hline Nonlinear coefficient $\eta$ & $742 \mathrm{~W}-2$ \\
Launch power per channel $P$ & $-1 \mathrm{dBm}$ \\
\hline \hline
\end{tabular}

processing (DSP) used after the mapper (i.e., pulse shaping, polarization multiplexing, filtering, electro-optical conversion, etc.), the physical channel (the fiber and amplifiers), and the receiver DSP (optical-to-electrical conversion, filtering, equalization, matched filtering, etc.). This channel is modeled using a dual-polarization, discrete-time, memoryless, additive white Gaussian noise (AWGN) channel.

For each polarization, and at each discrete time $k=$ $1,2, \ldots, n, Y_{k}=X_{k}+Z_{k}$, where $X_{k}$ is the transmitted symbol, $Z_{k}$ are independent, zero-mean, circularly symmetric, complex Gaussian random variables, and $n$ is the blocklength. This GN channel model characterizes optically-amplified links dominated by amplified spontaneous emission noise where chromatic dispersion and polarization mode dispersion are perfectly compensated. In this model, the power-dependent nonlinearities in the presence of sufficient dispersion are treated as an additional source of AWGN.

The symbol SNR for a light path with $N_{\mathrm{s}}$ spans is calculated as

$$
\mathrm{SNR}=\frac{P}{N_{s}\left(P_{\mathrm{ASE}}+\eta P^{3}\right)}
$$

where $P$ is the launch power per channel, $P_{\mathrm{ASE}}$ is the ASE noise added after each span (in the $32 \mathrm{GHz}$ signal bandwidth), $\eta$ is the nonlinear coefficient (per span).
The nonlinear interference is taken as that on the worst case central DWDM channel, i.e., we assumed that all the links were fully loaded with DWDM channels. The nonlinear coefficient $\eta$ is calculated using the incoherent GN model of nonlinear interference as defined in [33], SPM is assumed to be ideally compensated, and the ROADM nodes were assumed lossless. Using the parameters in Table I, we obtain $\eta \approx 742 \mathrm{~W}^{-2}$. The launch power that maximizes the SNR in (4) is found to be equal to [33, eq. (29)] $\sqrt[3]{P_{\mathrm{ASE}} /(2 \eta)}=-1 \mathrm{dBm}$. A summary of the parameters discussed in this section is given in Table I.

\section{Performance Metric: Network Throughput}

Throughout this paper, the performance metric under consideration is the total network throughput, which we define below and denote by $\Theta$. The network throughput as we define it, represents the total traffic transported by the network that satisfies a given traffic demand.

The physical network topology is assumed to have $N$ nodes, which we denote by the set $\mathcal{N} \triangleq\{1, \ldots, N\}$. We assume that there are $L_{s, d}$ available light paths connecting any two distinct nodes $s, d \in \mathcal{N}\left(L_{s, s}=0\right)$. We also denote the available capacity of the $l$ th light path by $C_{s, d}^{(l)}, l=1,2, \ldots, L_{s, d}$. The total available capacity between nodes $s$ and $d$ is then given by

$$
C_{s, d}=\sum_{l=1}^{L_{s, d}} C_{s, d}^{(l)}
$$

As discussed in Section II-C, in this paper we consider physical nonlinear impairments, and thus, the values of $C_{s, d}^{(l)}$ are different for different combinations of $s, d$ and $l$ in a given network. Consider for example the simple network topology in Fig. 5(a), where the different light paths are shown with different colors. In this example we assume nodes 1 and 2 are connected through 3 light paths $\left(L_{1,2}=3\right)$, and that they travel through different routes (green and orange). The nodes pairs $(1,3)$ and $(2,3)$ are connected through one light path only $\left(L_{1,3}=1\right.$ and $L_{3,2}=1$ ).

Furthermore, we assume different light paths have different capacities. Consider for example the following values:

$$
C_{1,2}^{(1)}=C_{1,2}^{(2)}=15, \quad C_{1,2}^{(3)}=5
$$


(a) Physical Network

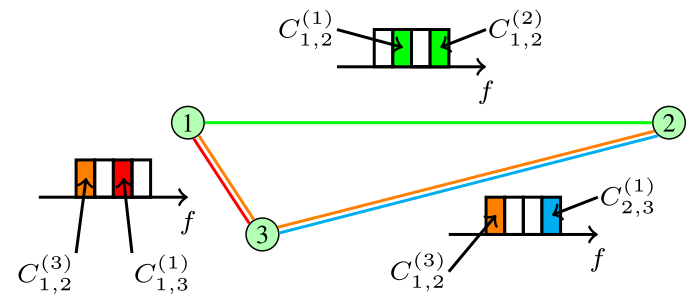

(b) Logical Network

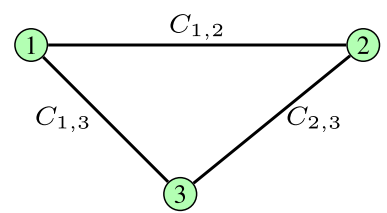

Fig. 5. Schematic representation of a (a) three-node physical network topology and (b) the corresponding logical network. In the physical network, different colors represent different light paths; for this network, $L_{1,2}=3, L_{1,3}=1$ and $L_{3,2}=1$.

and

$$
C_{1,3}^{(1)}=50, \quad C_{2,3}^{(1)}=15
$$

which leads to the following available capacity between nodes:

$$
C_{1,2}=35, \quad C_{1,3}=50, \quad C_{2,3}=15 .
$$

These available capacity between nodes can be associated to the links in a logical network, as shown in Fig. 5(b).

Once the capacities of the links in the logical network $C_{s, d}$ have been found, the network throughput could be defined as the sum of these capacities (i.e., $\sum_{s, d \in \mathcal{N}} C_{s, d}$ ). In this paper, however, we consider a given traffic demand that needs to be satisfied, and thus, the network throughput should be defined considering this constraint.

The traffic demand is defined by a normalized traffic matrix $\boldsymbol{T}$, whose entries $T_{s, d}$ with $s, d \in \mathcal{N}$, are normalized such that

$$
\sum_{s=1}^{N} \sum_{d=1}^{N} T_{s, d}=1 .
$$

We assume connectivity between all pairs of nodes is required $\left(0<T_{s, d}<1, s \neq d\right)$ and that $T_{s, d}=0$ if $s=d$. Under these constraints, the network throughput $\Theta$ is defined as

$$
\Theta \triangleq \min _{\substack{s, d \in \mathcal{N} \\ d \neq s}} \frac{C_{s, d}}{T_{s, d}} .
$$

To clarify the definition in (10), consider again the network in Fig. 5 with available capacities given by (8) and the following normalized traffic matrix:

$$
\boldsymbol{T}=\frac{1}{200}\left[\begin{array}{ccc}
0 & 35 & 50 \\
35 & 0 & 15 \\
50 & 15 & 0
\end{array}\right]
$$

In this case, the network throughput in (10) gives

$$
\Theta=\min \{200,200, \ldots, 200\}=200,
$$

which coincides with the sum of the available capacities $\left(\sum_{s, d \in \mathcal{N}} C_{s, d}=200\right)$. This is due to the fact that the normalized traffic matrix in (11) is matched to the available capacities in the logical network.

Consider now the following normalized traffic matrix:

$$
\boldsymbol{T}=\frac{1}{200}\left[\begin{array}{ccc}
0 & 55 & 5 \\
55 & 0 & 40 \\
5 & 40 & 0
\end{array}\right]
$$

which requires very little traffic between nodes 1 and 3 (which has large available capacity, $\left.C_{1,3}=50\right)$. In this case, the network throughput in (10) gives

$$
\Theta=\min \{127.2,2000,75\}=75
$$

which is less than half of the sum of the total available capacities $\sum_{s, d \in \mathcal{N}} C_{s, d}=200$. The intuition behind the result in (14) is that although large amounts of traffic can be transported between nodes 1 and 3 , only very little is required by the normalized traffic matrix in (13). This causes underutilization of the resources, which in turn gives a lower network throughput. Nevertheless, if a given normalized traffic matrix is to be fulfilled, and this matrix does not perfectly match the logical network, the network throughput definition in (10) should be used.

Although the network throughput definition (10) is general in the sense that it considers arbitrary normalized traffic matrices, in this paper we consider a uniform normalized traffic matrix, i.e.,

$$
T_{s, d}=\left\{\begin{array}{ll}
\frac{1}{N(N-1)}, & s \neq d \\
0, & s=d
\end{array} .\right.
$$

The network throughput in this case reduces to

$$
\Theta=N(N-1) \min _{\substack{s, d \in \mathcal{N} \\ d \neq s}} C_{s, d} .
$$

For the example in Fig. 5 with available capacities given by (8), the uniform normalized traffic matrix is given by

$$
\boldsymbol{T}=\frac{1}{6}\left[\begin{array}{lll}
0 & 1 & 1 \\
1 & 0 & 1 \\
1 & 1 & 0
\end{array}\right]
$$

and the network throughput is

$$
\Theta=\min \{210,300,90\}=90 .
$$

The matrix $\boldsymbol{T}$ in (17) does not match the logical network, and thus, the obtained throughput is again below the sum of the available capacities. It should be noted, however, that a network throughput of 200 (the sum of the available capacities) cannot be transported through the network while at the same time fulfilling the normalized traffic matrix in (17). Therefore, from now on, we only consider $\Theta$ in (16). 


\section{Optimal Modulation AND FEC OH}

In this section, we describe the selection of optimal FEC OH and modulation format from an information theory viewpoint. We first consider the ideal case of continuous constellations and then move to the case of modulation with discrete number of constellation points. The routing and wavelength assignment problem and the maximum network throughput are also discussed in this section.

\section{A. Channel Capacity}

The capacity of the AWGN channel (in [bit/sym]) under an average power constraint is

$$
C=2 \log _{2}(1+\mathrm{SNR})
$$

where the pre-log factor of 2 takes into account the two polarizations. The value of $C$ represents the maximum number of information bits per symbol that can be reliably transmitted through an AWGN channel.

The capacity in (19) is achieved when the transmitted symbols are chosen from a zero-mean Gaussian distribution. In practice, however, the modulation has $M$ discrete levels, which reduces the achievable transmission rates. This case is studied in the next section.

\section{B. Achievable Rates for Discrete Constellations}

From an information theory point of view, the optimal code rate and constellation size can be chosen from the mutual information (MI) between the input and output symbols of the channel, usually denoted by $I(X ; Y)$. Although the MI can be defined between any two random variables $X$ and $Y$, it has a very important operational meaning in the context of data transmission: the MI $I(X ; Y)$ is an achievable rate for an optimal receiver. ${ }^{4} \mathrm{MI}$ curves for square QAM constellations indicate that, regardless of the SNR, in order to maximize the SE, the densest available constellation should always be used and the code rate chosen between 0 and $1 .^{5}$ This has been shown, e.g., in [7, Fig. 4.3], [15, Fig. 1], [33, Fig. 11].

The MI is not an achievable rate for the two receiver structures we consider in this paper (see Fig. 1). The first receiver in Fig. 1 is suboptimal because it makes HDs on the symbols (and thus, information is lost). The second receiver is suboptimal because the LLR calculation ignores the dependency of the bits within a symbol (i.e., $L_{q}$ in (3) does not depend on $B_{l}$ for $l \neq q$ ). In this paper we consider two different achievable rates, one for each of these receiver structures.

For the case of a BW receiver with HDs, Shannon's coding theorem states that error-free transmission is possible when

\footnotetext{
${ }^{4}$ In information theory, a transmission rate $R$ is said to be achievable if there exist an encoder with rate $R$ and a (possibly very complex) decoder, such that the error probability after decoding vanishes as $n \rightarrow \infty$.

${ }^{5}$ This is difficult to realize in practice as high-order modulation formats at low SNRs are hard to implement. The DSP limitations in the choice of high-order modulation formats have been recently discussed in [45].
}

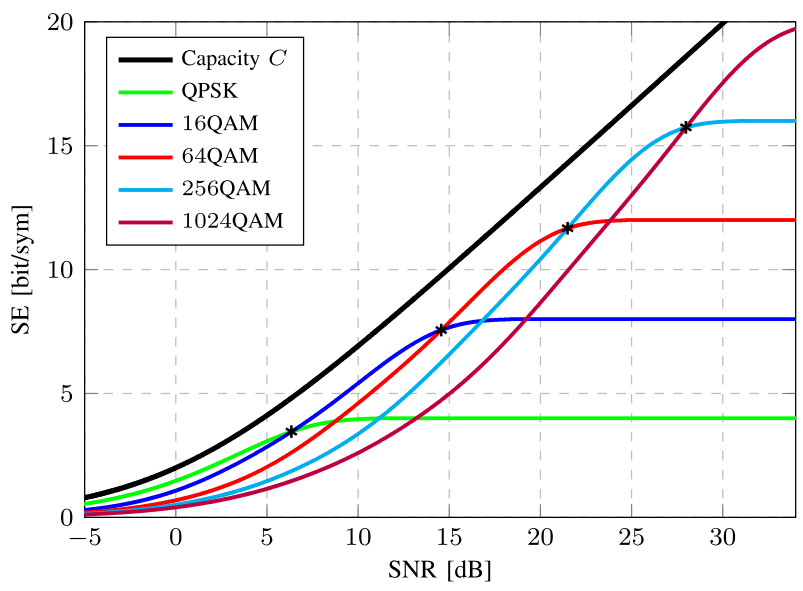

Fig. 6. Achievable rates for a BW receiver with HD-FEC. The black asterisks show the SNR values where the modulation size should be changed. The channel capacity in (19) is shown for comparison.

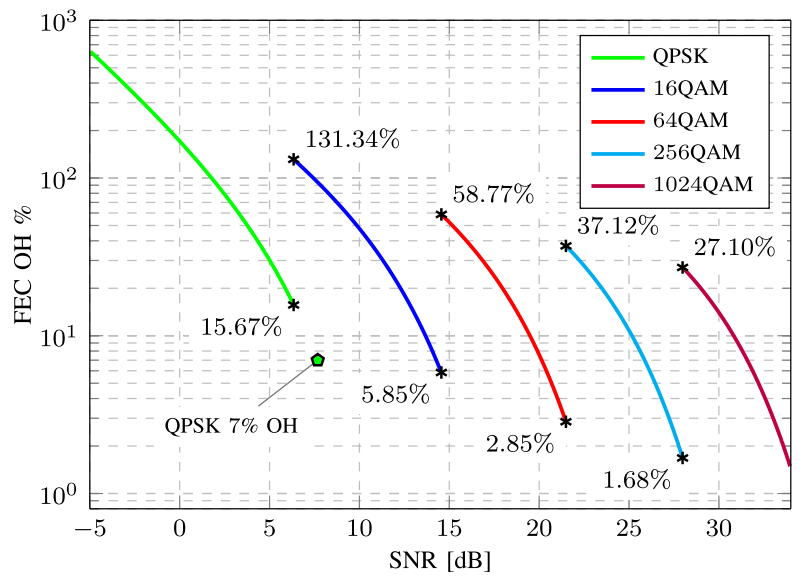

Fig. 7. OHs obtained from the achievable rates in Fig. 6 for a BW receiver with HD-FEC. The black asterisks show the SNR values where the modulation size should be changed and the corresponding FEC OH. The channel capacity in (19) is shown for comparison. The SNR required for QPSK with 7\% FEC $\mathrm{OH}$ is also shown with a green pentagon.

$n \rightarrow \infty$ if the rate of the encoder fulfills [16, eq. (5)] [46, eq. (8)]

$$
R_{c} \leq \frac{I(B ; \hat{B})}{m}=1-H_{b}(\mathrm{BER})
$$

where $I(B ; \hat{B})$ is the MI between the transmitted and received code bits (see Fig. 1). In (20), $H_{b}(p)=-p \log _{2} p-$ $(1-p) \log _{2}(1-p)$ is the binary entropy function and BER is the average pre-FEC BER (across $m$ bit positions).

The values of $I(B ; \hat{B})$ for $M=4,16, \ldots, 1024$ are shown in Fig. 6, where the BER was calculated using [47, Th. 2]. The key difference between the achievable rates $I(B ; \hat{B})$ and $I(X ; Y)$ is that the former cross each other for different values of $M$ (see the asterisks in Fig. 6). An important consequence of crossing achievable rate curves is that the theoretically optimal choice of $R_{c}$ and $M$ is not straightforward. For a BW receiver with HDFEC we consider here, QPSK should be used for SNRs below $\mathrm{SNR} \leq 5.8 \mathrm{~dB}, 16 \mathrm{QAM}$ for $5.8 \leq \mathrm{SNR} \leq 14 \mathrm{~dB}$, etc. The corresponding FEC OHs obtained via (2) are shown in Fig. 7. This figure also shows the optimum minimum and maximum $\mathrm{OH}$ 


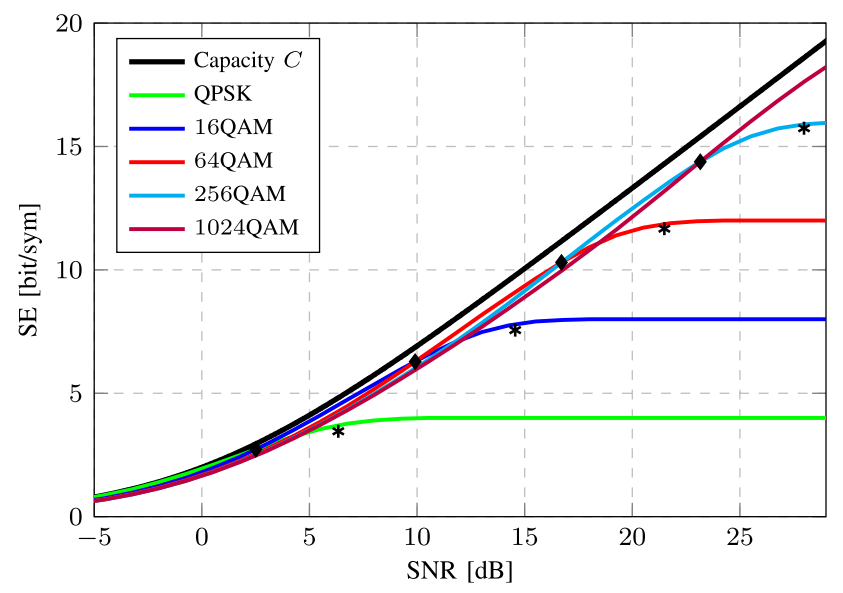

Fig. 8. Achievable rates (GMI) for a BW receiver with SD-FEC. The black diamonds show the SNR values where the modulation size should be changed. The channel capacity in (19) and the crossing points of the achievable rates for HD-FEC (asterisks) taken from Fig. 6 are also shown for comparison.

values for each modulation format as well as the SNR required for QPSK with 7\% FEC OH (green pentagon).

When the BW receiver operates with SDs, and if the code bits are independent, an achievable rate is given by the GMI [7, eq. (4.55)], [10, eq. (13)], [11, eq. (25)]

$$
\mathrm{GMI}=\sum_{k=1}^{m} I\left(B_{k} ; Y\right)=\sum_{k=1}^{m} I\left(B_{k} ; L_{k}\right)
$$

where the second equality holds if the LLRs are calculated via (3) and where $I\left(B_{k} ; L_{k}\right)$ is the MI between the code bits and LLRs before FEC decoding. The three achievable rates considered above (MI, (20) and (21)) are schematically shown in Fig. 1.

Fig. 8 shows the GMI in (21) for different constellations. Similarly to the achievable rates for HD-FEC, the GMI curves cross each other (see black diamonds). ${ }^{6}$ Although this effect is less noticeable, the theoretical implications are the same: different SNRs call for different modulation sizes and FEC OH. In Fig. 8, we also show (with asterisks) the crossing points of the achievable rates for HD-FEC taken from Fig. 6. We do this to emphasize that if SD-FEC is considered instead of HDFEC, different (higher) SNR thresholds are obtained. This is also visible in Fig. 9, where the optimum FEC OHs for SDFEC are shown. The results in Figs. 8 and 9 have been recently experimentally studied in [50] and [51].

\section{Routing and Wavelength Assignment Problem}

The routing and wavelength assignment problem is solved numerically as an integer linear programming (ILP) problem as described in [40, Sec. 4.1]. In particular, we maximize the network throughput in (16) (i.e., under a uniform traffic demand), where $C_{s, d}^{(l)}$ are assumed to be given by the capacity

\footnotetext{
${ }^{6} \mathrm{We}$ again emphasize that this is only due to the fact a suboptimal receiver is considered. MI curves, on the other hand, do not cross each other for $M$ QAM constellations, which has been known for many years (see, e.g., [48, Fig. 2], [49, Fig. 1]).
}

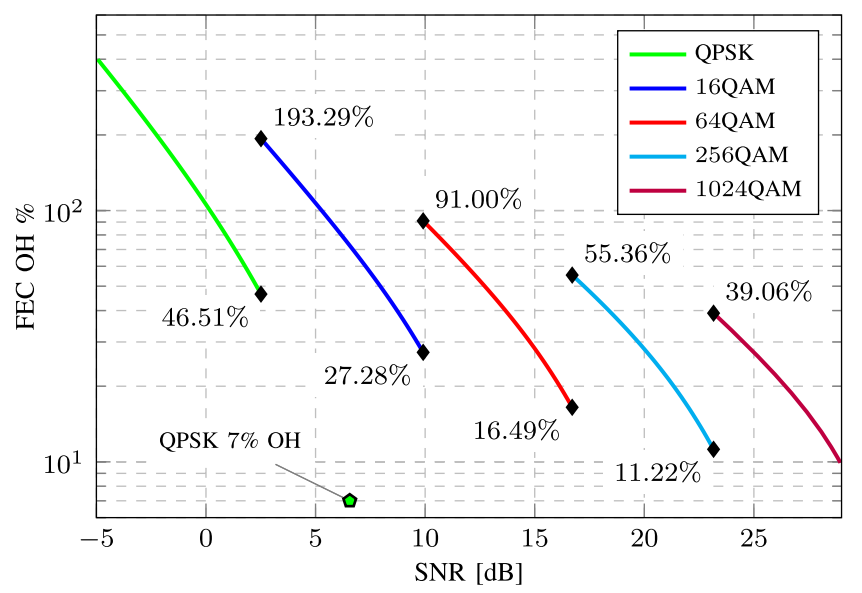

Fig. 9. OHs obtained from the achievable rates in Fig. 8 for a BW receiver with SD-FEC. The black diamonds show the SNR values where the modulation size should be changed and the corresponding FEC OH. The channel capacity in (19) is shown for comparison. The SNR required for QPSK with 7\% FEC $\mathrm{OH}\left(\mathrm{SNR}_{7 \%}\right)$ is also shown with a green pentagon.

function $C$ in (19). The ILP solution provides the number of active light paths and their routes between each node pair. From this, the total number of active light paths in the network is obtained. This solution also provides the SNR of each active light path.

The SNR values obtained by solving the ILP problem for the three network topologies in Figs. 2-4 are shown in Fig. 10. The vertical bars show the number of transceivers that need to be installed to maximize the network throughput. The number of (two-way) transceivers for the DTG, NSF, and DTG networks are 1230,1094 , and 570 , respectively.

The vertical bars in Fig. 10 can be interpreted as the distribution of SNR across the network. By comparing these distributions, it is clear that the average SNRs across the network decreases as the size of the network increases. This is due to the presence of long links in continental and transcontinental networks (NSF and GB4). The SNR distributions in Fig. 10 also show that the spread of the SNR values is much larger for large networks. While for the DTG network, the variation in SNR is about $10 \mathrm{~dB}$, for the GB4 network, this variation is about $20 \mathrm{~dB}$.

Once the SNR values for the active light paths are found, the maximum network throughput can be calculated via (16). In particular, the SNRs of the light paths shown in Fig. 10 are first grouped for each source destination pair. Then, the SNRs are "mapped" to throughputs via (19), and the value of $C_{s, d}$ is obtained via (5). The resulting throughputs are 524, 278, and $88 \mathrm{~Tb} / \mathrm{s}$, for the DTG, NSF, and GB4 networks, respectively. These throughput values are shown in the first columns of Table II together with the number of transceivers for each network.

\section{Ideal FEC}

The results in the previous section assume all the transceivers can achieve the capacity of the AWGN channel. This is never 

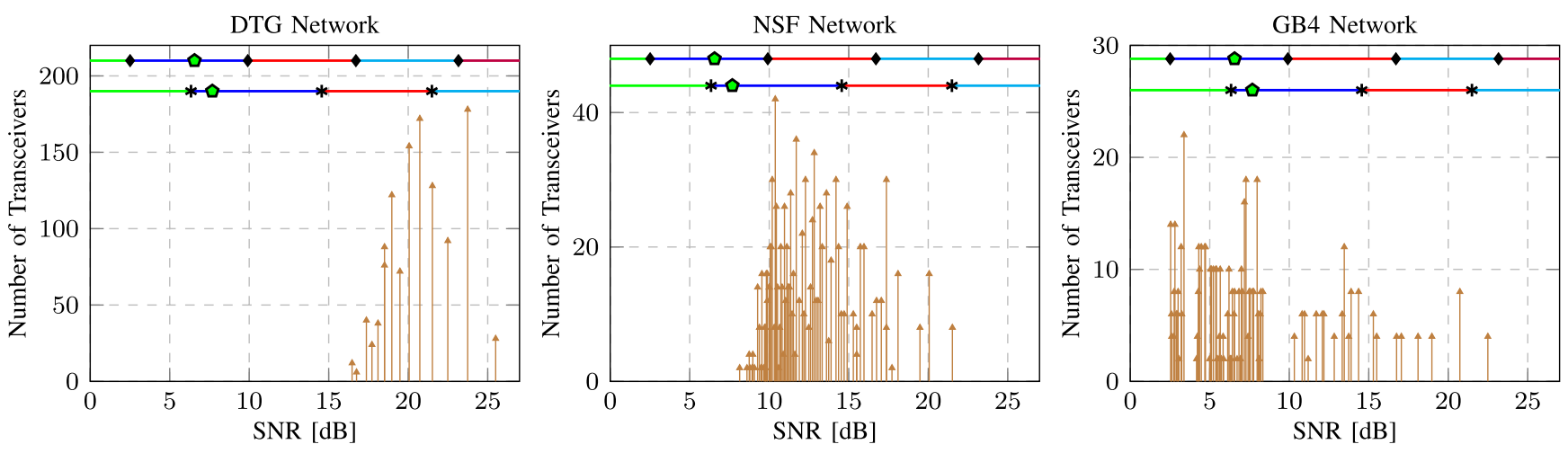

Fig. 10. Number of transceivers (vertical lines with triangles at the top) for all SNR values for the three network topologies in Figs. 2-4. These values are obtained by assuming all transceivers can achieve the capacity $C$ in (19). The horizontal lines show the SNR ranges in which different formats should be used. As in Figs. 7 and 9, asterisks and diamonds represent the SNR thresholds for HD- and SD-FEC, resp. The SNR thresholds for QPSK with 7\% OH are also shown with green pentagons.

TABLE II

OPTIMUM NUMBER OF TRANSCEIVERS AND NETWORK THROUGHPUT $\Theta$ (IN TB/S) UNDER IDEALISTIC ASSUMPTIONS ON CODING AND MODULATION

\begin{tabular}{|c|c|c|c|c|c|}
\hline \multirow[b]{2}{*}{ Network } & \multirow[b]{2}{*}{$\begin{array}{c}\text { Nodes } \\
N\end{array}$} & \multirow[b]{2}{*}{ Transceivers } & \multicolumn{3}{|c|}{ Network Throughput $\Theta$ (in $[\mathrm{Tb} / \mathrm{s}])$} \\
\hline & & & $\begin{array}{c}\text { Maximum } \\
\text { (Capacity-based) }\end{array}$ & $\begin{array}{l}\text { Ideal } \\
\text { HD-FEC }\end{array}$ & $\begin{array}{c}\text { Ideal } \\
\text { SD-FEC }\end{array}$ \\
\hline DTG & 9 & 1230 & 524 & 431 & 489 \\
\hline NSFNET & 14 & 1094 & 278 & 217 & 255 \\
\hline GB4 & 12 & 570 & 88 & 64 & 81 \\
\hline
\end{tabular}

the case in practice as it requires the use of continuous constellations. In this section we consider the case where all transceivers can choose any of the MQAM constellations considered in this paper. Although more practically relevant, we assume the code rate $R_{c}$ can be adjusted continuously, which is again never the case in practice. Nevertheless, the results in this section can be used to estimate the penalty caused by the use of discrete (and square) constellations.

The selection of code rate and modulation format is assumed to be based on the achievable rates discussed in Section IV-B. This idea is shown schematically in Fig. 10, where horizontal lines with different colors are included. These lines show the SNR ranges where different modulation formats should be used (lines with diamonds for SD-FEC and lines with stars for HDFEC) and are obtained from Figs. 9 and 7.

To obtain the throughput achieved by ideal HD- and SD-FEC, we again use (16) and follow similar steps to those used in Section IV-C. Namely, the SNRs of the light paths (shown in Fig. 10 and obtained from the ILP solution) are first grouped for each source destination pair and the SNRs are then "mapped" to throughputs via the achievable rates discussed in Section IV-B. The network throughputs obtained for HD-FEC, are 431, 217, and $64 \mathrm{~Tb} / \mathrm{s}$, for the DTG, NSF, and GB4 networks, respectively. For SD-FEC, these values become 489, 255, and $81 \mathrm{~Tb} / \mathrm{s}$. These throughput results are shown in the last two columns of Table II.
The results in Table II show that, when compared to the maximum throughput obtained via the AWGN capacity assumption (fourth column in Table II), the use of ideal SD-FEC causes a relative throughput decrease of $9 \%, 8 \%$, and $7 \%$, for the DTG, NSF, and GB4 networks, respectively. This indicates a relatively constant loss across different network topologies. On the other hand, the use of ideal HD-FEC causes relative losses of $18 \%, 22 \%$, and $27 \%$. These results show an increasing loss as the network size increases, which in turn shows the importance on considering SD-FEC for large networks. We conjecture that these increasing losses are due to the different shape of the "envelopes" of the crossing achievable rates in Figs. 6 and 8.

When compared to SD-FEC, HD-FEC codes are typically low complexity and low latency. On the other hand, for the same SNR, HD-FEC codes need higher OH to operate error free, which causes a throughput loss. The relative throughput losses are approximately $12 \%, 15 \%$, and $20 \%$ for the DTG, NSF, and GB4 networks, respectively. This indicates that low complexity and latency can be traded by a 10-20\% loss in throughput and that the use SD-FEC becomes more and more important as the network size increases.

\section{PRACTICAL SCHEMES}

Due to the continuous code rate assumption, the throughputs in the last two columns of Table II are only upper bounds that cannot be achieved in practice. In this section we discuss practically relevant alternatives.

\section{A. QPSK With $7 \%$ FEC OH}

Probably the simplest (and most popular) alternative in terms of coding and modulation for an optical network is to consider QPSK and a fixed FEC OH of 7\% across the network. In this case, if the SNR of a given light path is below the required SNR for QPSK with 7\% OH, the light path will not be used. If the SNR is above the threshold, then the available throughput in the $l$ th light path $C_{s, d}^{(l)}$ will be given by the SE in (1) times the 
symbol rate. The total available throughput (in $[\mathrm{Tb} / \mathrm{s}]$ ) in (10) is then given by

$$
C_{s, d}^{(l)}= \begin{cases}\frac{0.128}{1.07}, & \text { if } \operatorname{SNR}_{s, d}^{(l)} \geq \mathrm{SNR}_{7 \%} \\ 0, & \text { otherwise }\end{cases}
$$

where $\mathrm{SNR}_{s, d}^{(l)}$ is the SNR of the $l$ th light path and $\mathrm{SNR}_{7 \%}$ is the SNR required for QPSK with 7\% $\mathrm{OH}$ (shown with green pentagons in Figs. 7, 9, and 10).

The total network throughput in (16) (in Tb/s) is then

$$
\Theta=N(N-1) \cdot \frac{0.128}{1.07} \min _{\substack{s, d \in \mathcal{N} \\ d \neq s}} \sum_{l=1}^{L_{s, d}} I_{\left[\mathrm{SNR}_{s, d}^{(l)} \geq \mathrm{SNR}_{7 \%}\right]}
$$

where $I_{[\nu]}$ is an indicator function, i.e., $I_{[\nu]}=1$ if $\nu$ is true, and $I_{[\nu]}=0$ otherwise.

The SNR threshold $\mathrm{SNR}_{7 \%}$ in (23) is different for HD- and SD-FEC, and thus, the resulting throughputs might also be different. However, for both the DTG and NSF networks, all the light path SNRs are above both thresholds, and thus, the total network throughput in (23) is

$$
\Theta=N(N-1) \cdot \frac{0.128}{1.07} \min _{\substack{s, d \in \mathcal{N} \\ d \neq s}}\left\{L_{s, d}\right\} .
$$

The minimum number of light paths for any source destination pair for the NTG and NSF networks are 14 and 4, respectively, which combined with the number of nodes in the network, give throughputs of 120 and $87 \mathrm{~Tb} / \mathrm{s}$.

For QPSK and 7\% FEC OH, SD offers a theoretical maximum sensitivity increase of about $1.15 \mathrm{~dB} .{ }^{7}$ However, for the DTG and NSF networks, there is no difference between HD and SD in terms of network throughput as the minimum SNR of all light paths is above the SNR threshold. This result highlights the fact that under these conditions and traffic assumptions, upgrading all transceivers from HD to SD FEC without changing the $\mathrm{OH}$ might not bring any benefit for small networks.

When the GB4 network is considered, however, most of the light paths are in fact below the SNR required for QPSK with 7\% $\mathrm{OH}$ (see Fig. 10). For this case, the network throughput given by (23) is in fact zero. This can be intuitively explained by the fact that there are node pairs that are very far apart, and thus, the uniform throughput constraint and full network connectivity cannot be satisfied.

\section{B. Single-Modulation Schemes}

As an alternative to the QPSK with 7\% FEC OH approach, in this section we consider two approaches, both of them based on the approach that only one modulation format should be implemented across the network.

The first scheme assumes all transceivers implement one modulation format and one code rate. We call this scheme

\footnotetext{
${ }^{7}$ This can be obtained by comparing the green pentagons for HD and SD FEC in Figs. 7 and 9.
}

$1 R_{c} 1 M$. In this scheme, the code rate is chosen such that the network throughput (based on achievable rates) is maximized. The results obtained for $1 R_{c} 1 M$ are shown in Fig. 11 with red triangles for HD-FEC and with blue triangles for SD-FEC. The top figure shows network throughput, while the bottom ones show optimum code rates. The different networks under consideration are shown from left to right. In the throughput results, we also include the ideal network throughputs in Table II (solid horizontal lines) as well as the results obtained with QPSK and 7\% FEC OH from Section V-A (green pentagons).

The results in Fig. 11 show that for HD-FEC and $1 R_{c} 1 M$, there is always a modulation format that is optimum: $M=64$ for DTG, $M=16$ for NSF, and $M=4$ for GB4. Nevertheless, for both HD- and SD-FEC, the gains obtained (with respect to QPSK with $7 \% \mathrm{OH}$ ) by using $1 R_{c} 1 M$ are quite large. Interestingly, the optimum code rates for HD-FEC and $1 R_{c} 1 M$ are $R_{c} \approx 0.8$ for DTG, $R_{c} \approx 0.62$ for NSF, and $R_{c} \approx 0.56$ for GB4 ( $24 \%, 61 \%$ and $79 \%$ FEC OH, respectively). This indicates a clear potential benefit of using large FEC OH when HD-FEC is used in a network context and where the modulation format is fixed across the network.

When it comes to $1 R_{c} 1 M$ with SD-FEC, the optimality of a given modulation format is less clear, as the throughput curves in this case do not have a clear peak. This is due to the fact that the crossings between the GMI curves is not as pronounced as the crossings for HD-FEC. Nevertheless, by observing the trend of the curves, a good compromise would be to choose the same modulation formats as for HD-FEC, i.e., $M=64$ for DTG, $M=16$ for NSF, and $M=4$ for GB4. In this case, the corresponding optimum code rates are $R_{c} \approx 0.89, R_{c} \approx 0.75$, and $R_{c} \approx 0.68$ (12\%, 33\% and $47 \%$ FEC OH, respectively). In general, the optimum code rates in this case are slightly higher than the ones for HD-FEC. The gains of SD-FEC over HD-FEC in this case are approximately $50 \mathrm{~Tb} / \mathrm{s}$ for the DTG and NSF networks. On the other hand, only small gains are observed for the GB4 network.

As mentioned before, the throughput for QPSK with 7\% FEC OH for the GB4 network is zero. On the other hand, for this network, $1 R_{c} 1 M$ gives throughputs of about $20 \mathrm{~Tb} / \mathrm{s}$. This is obtained by using QPSK and an increased $\mathrm{OH}$. This result highlights the need to consider high FEC OH in large networks.

The second scheme we consider in this section is called $2 R_{c} 1 M$. In this case, the transceivers operate with one modulation format but with two code rates. Again, the code rates are optimized so that the network throughput is maximized.

The results obtained for $2 R_{c} 1 M$ are shown in Fig. 11 with circles (red for HD-FEC and blue for SF-FEC). These results show that, regardless of the network under consideration, half of the gap between the ideal FEC limit (horizontal lines) and the throughput obtained by $1 R_{c} 1 M$ can be harvested by using an extra code rate. These results highlight the advantage of adapting the code rate to the variable channel conditions across the network. These results also show that for both SD- and HDFEC, the penalty with respect to the ideal case is about $85 \%$, $75 \%$, and $53-56 \%$, for DTG, NSF, and GB4, respectively. 


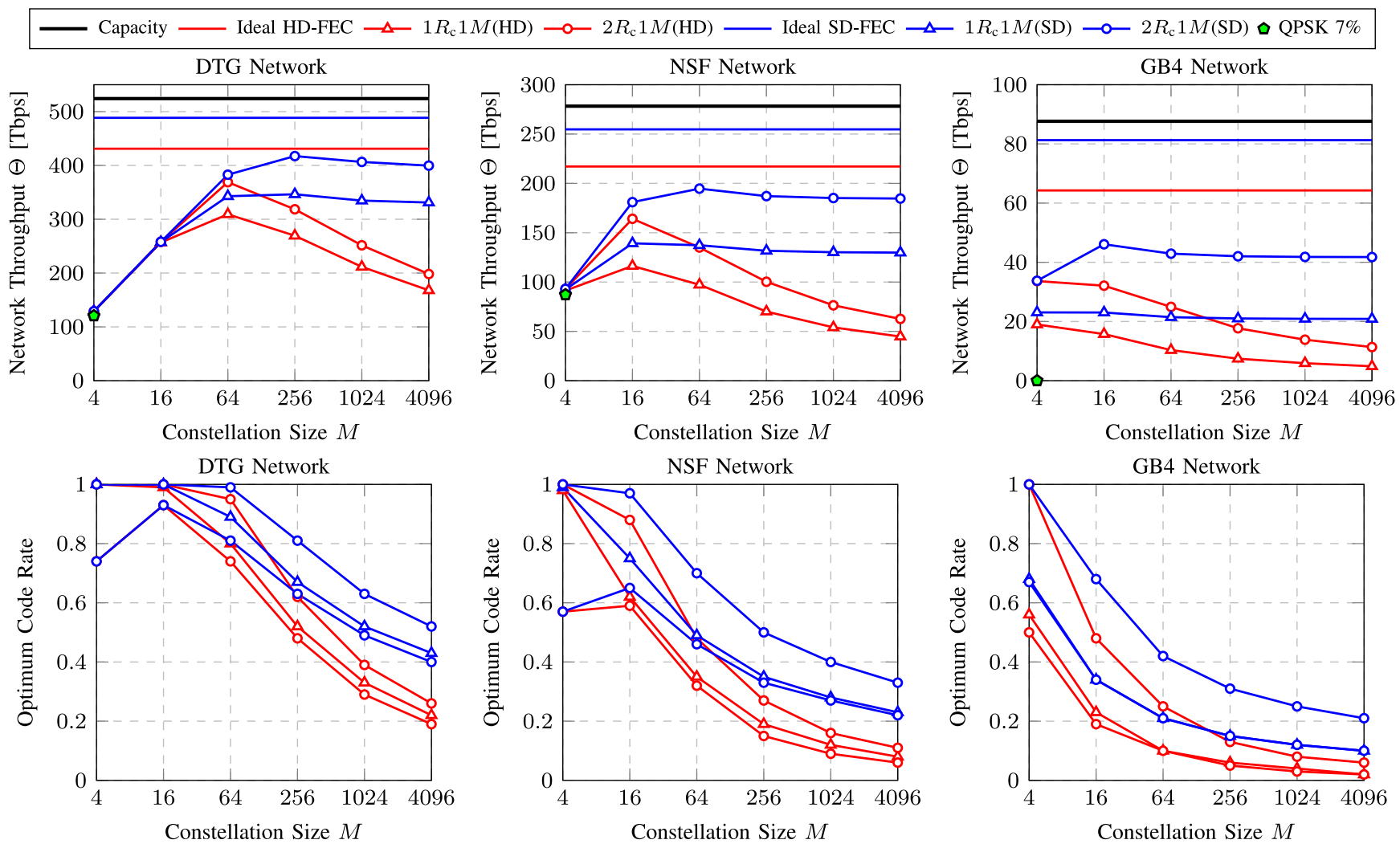

Fig. 11. Throughput (top row) and optimum code rate (bottom row) for one $M$ schemes, for both HD-FEC (red) and SD-FEC (blue), and for the three network topologies in Figs. 2-4. The throughput results obtained with QPSK and 7\% FEC OH in Section V-A are also shown with green pentagons.

This suggests that a good complexity-performance tradeoff can be obtained by using one modulation format and two code rates for national and continental networks, while for transcontinental networks, more code rates should be used.

\section{Variable-Modulation Schemes}

In this section we consider a design strategy where the modulation format is variable. In particular, we assume all the transceivers can choose any modulation format in between QPSK and a given maximum value of $M$, which we denote by $\hat{M}$. For example, if $\hat{M}=64$, then the transceivers can choose from $M=4, M=16$ and $M=64$.

The first scheme we consider assumes only one code rate is implemented across the network, and that the code rate is optimized to maximize the network throughput. We denote this scheme $1 R_{c} \operatorname{Var} M$. The results obtained using this scheme are shown in Fig. 12 (squares) and indicate that, in general, constellation sizes beyond 256QAM give little throughput increases (the throughput curves flatten out for large values of $\hat{M}$ ).

Figs. 13 and 14 show the percentage of transceivers using different modulation formats for $1 R_{c} \operatorname{Var} M$, for HD- and SD-FEC, respectively, and for $\hat{M}=16,64,256$. The value in the middle of each chart is the total throughput obtained. The general trend in these results is that QPSK (green) is only useful in the very large network (GB4). These results also show that, considering 256QAM (light blue) gives very little throughput increases for the NSF and GB4 networks, for both HD- and SD-FEC. On the other hand, 256QAM gives a relevant throughput increase for the DTG network.

To compare the throughput contribution of different modulation formats for $1 R_{c} \operatorname{Var} M$, we show in Fig. 15 the obtained throughputs for both HD- and SD-FEC. Apart from showing the relative contributions, this figure also shows how the contributions change when SD-FEC is considered instead of HDFEC. The throughput gains due to SD-FEC are also clearly visible.

The results in Figs. $12-15$ show that $1 R_{c} \operatorname{Var} M$ gives similar throughput results to those obtained by $2 R_{c} 1 M$ (two code rates and one modulation format) shown in Fig. 11. This indicates that similar (large) gains, can be obtained by having either multiple code rates or multiple modulation formats.

The second scheme we consider in this section is one where two code rates are implemented across the network (and the modulation can be varied too). We call this scheme $2 R_{c} \operatorname{Var} M$. The results are shown with plus-circles in Fig. 12. These results indicate that including a second code rate gives a clear advantage with respect to $1 R_{c} \operatorname{Var} M$ (squares). This gain is particularly visible for large values of $\hat{M}$ and large networks. In particular, for the GB4 network and $\hat{M}=256$, the gains are approximately $15 \mathrm{~Tb} / \mathrm{s}$ for both HD- and SD-FEC.

We conclude this section by comparing the optimal code rates of two-rate versus the one-rate schemes. In particular, we observe from Figs. 11 and 12 that the smallest code rate for 


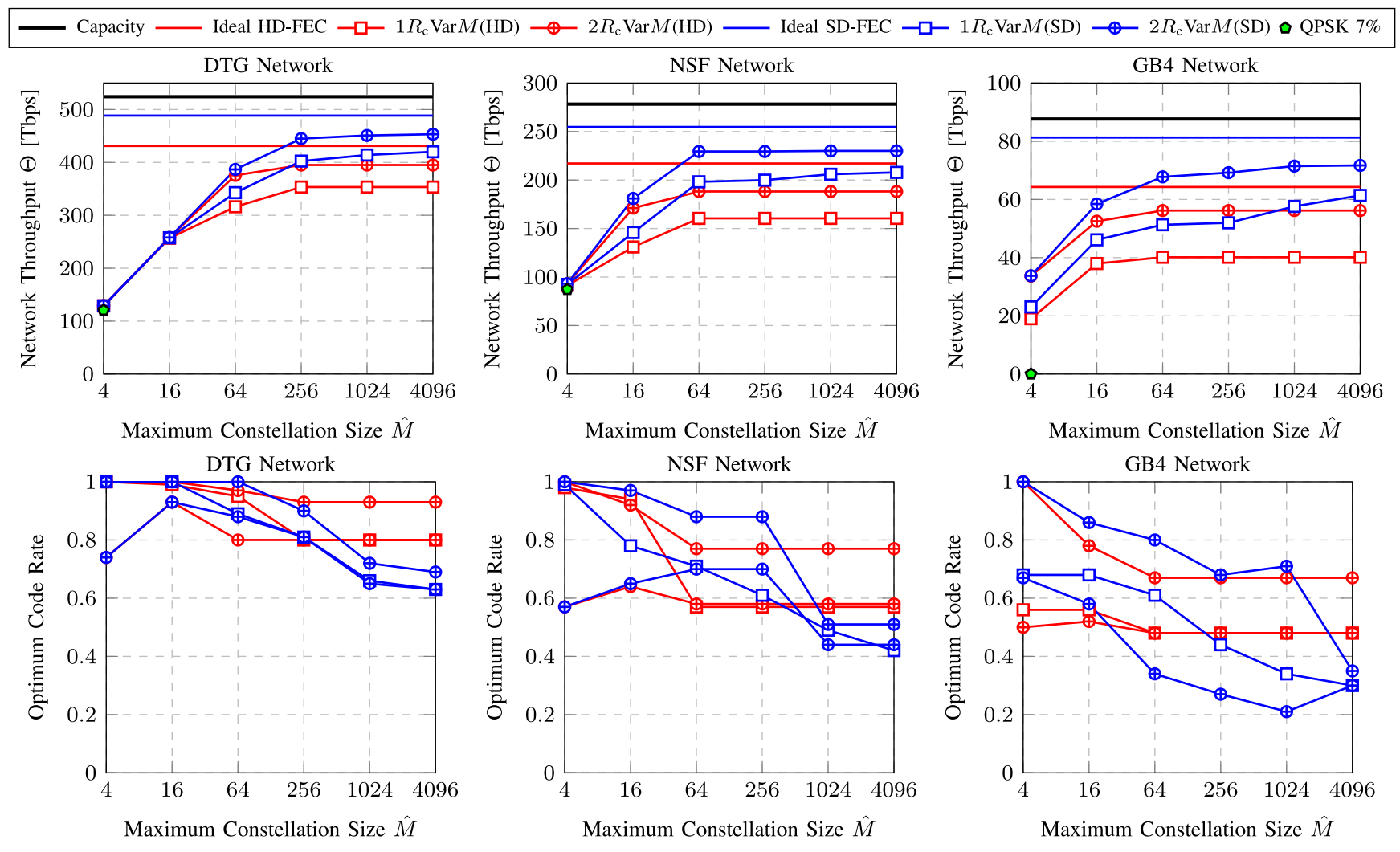

Fig. 12. Throughput (top row) and optimum code rate (bottom row) for variable $M$ schemes, for both HD-FEC (red) and SD-FEC (blue), and for the three network topologies in Figs. 2-4. The throughput results obtained with QPSK and 7\% FEC OH in Section V-A are also shown with green pentagons.

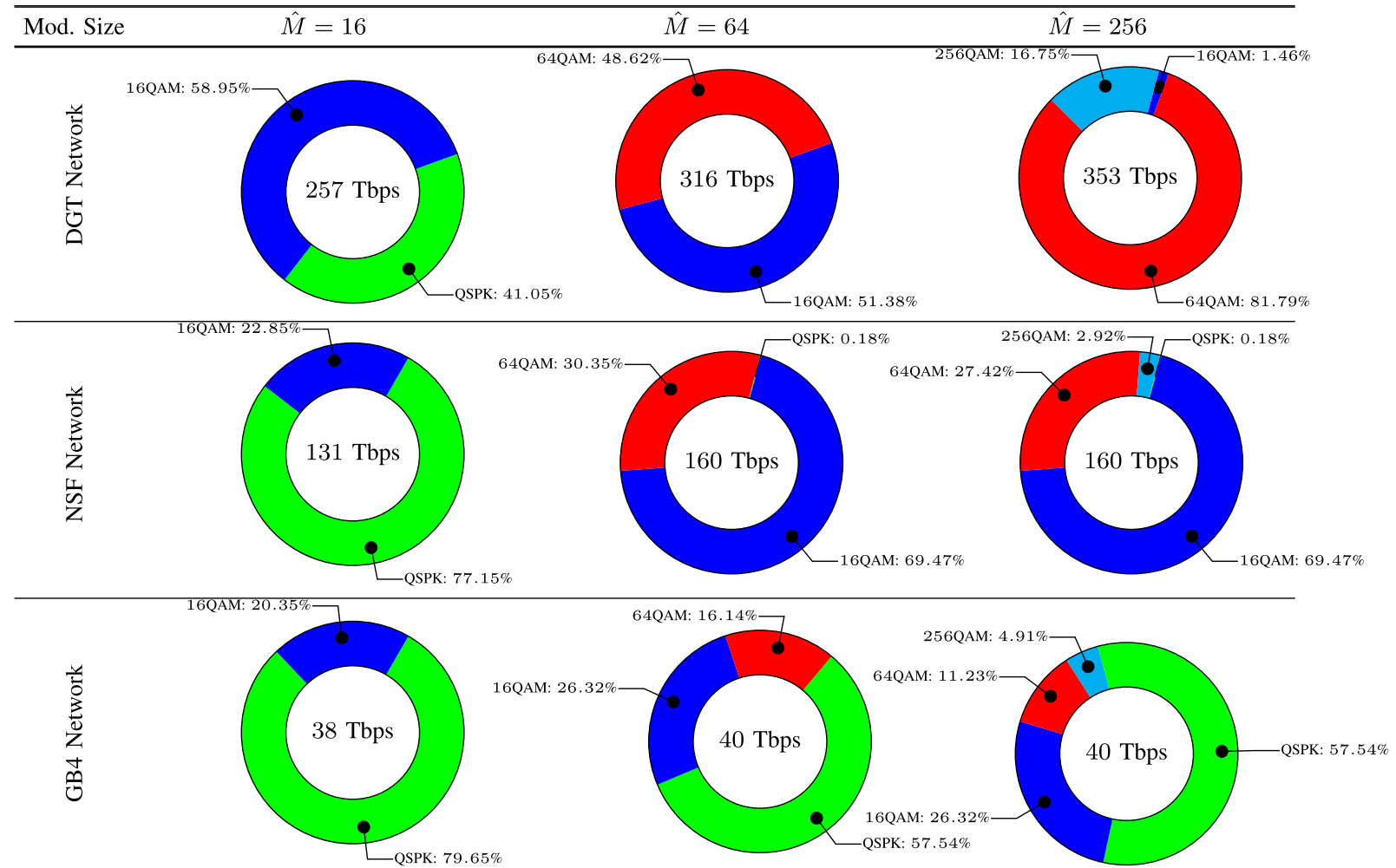

Fig. 13. Percentage of transceivers using different modulation formats for the optimal HD-FEC solution (for $1 R_{c}$ Var $M$ ) and the three network topologies. The results are shown for different maximum constellation sizes $\hat{M}$. 


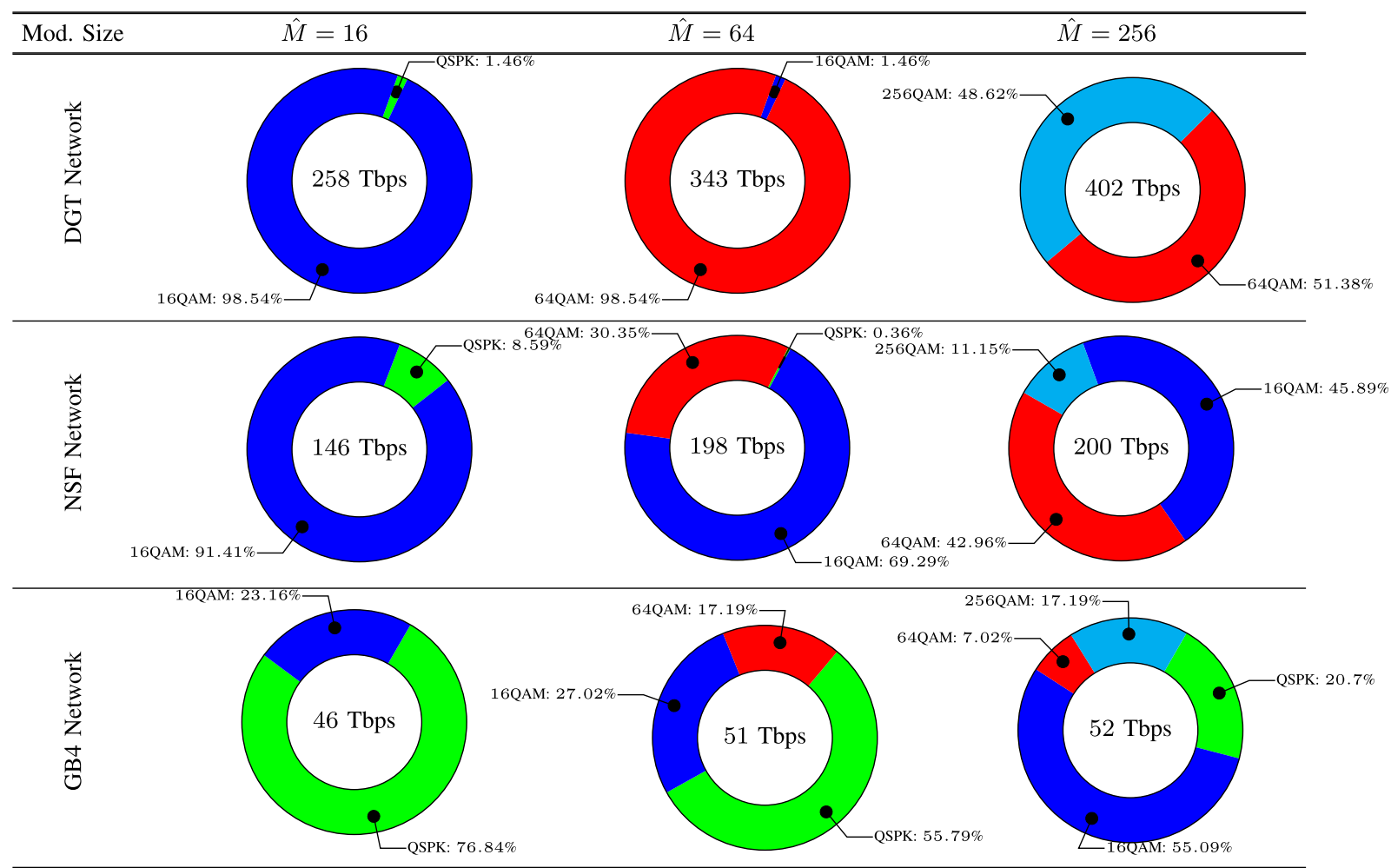

Fig. 14. Percentage of transceivers using different modulation formats for the optimal SD-FEC solution (for $1 R_{c} \operatorname{Var} M$ ) and the three network topologies. The results are shown for different maximum constellation sizes $\hat{M}$.

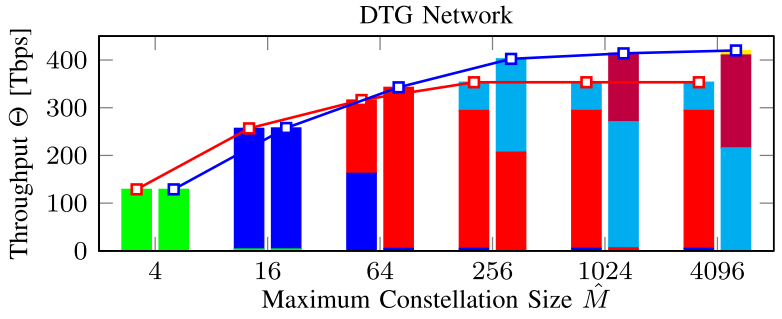

NSF Network

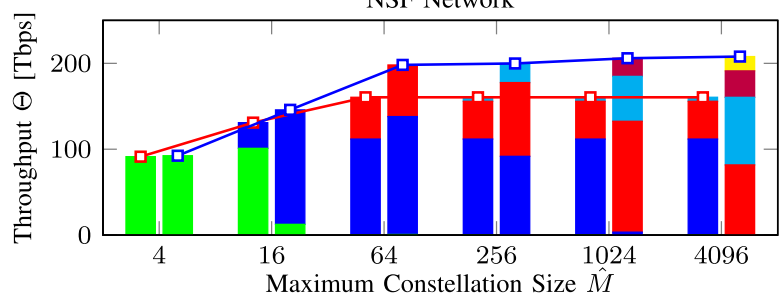

GB4 Network

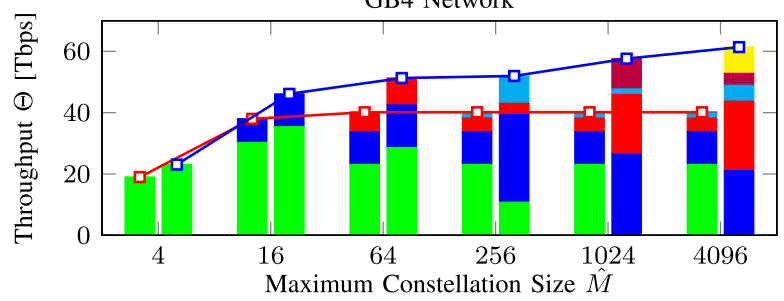

Fig. 15. Network throughput for $1 R_{c} \operatorname{Var} M$ for HD-FEC (red lines with squares) and SD-FEC (blue lines with squares) as a function of the maximum constellation size for the three network topologies in Figs. 2-4. The colors represent the throughput contribution from the different modulation sizes. The lines with markers are the network throughput shown in Fig. 12. the two-rate schemes is always quite close to the optimal code rate of the corresponding one-rate scheme. The intuition behind this is that when the transmitters are equipped with two code rates, one low code rate can be used for the worst performing connection, while the other rate is used to increase the overall network throughput.

\section{CONCLUSION}

Optimal constellation sizes and FEC OHs for optical networks were studied. Joint optimization of the constellation and FEC OHs was shown to yield large gains in terms of overall network throughput. The optimal values were shown to be dependent on the SNR distributions within a network. Two code rates and a single constellation (which varies as a function of the network size) gave a good throughput-complexity tradeoff.

In this paper, we studied the problem from the point of view of largest achievable rates. Practical FEC implementations, however, will operate a few decibels (or fractions of decibels) away from these achievable rates. Extra penalties are also expected from the use of high-order modulation formats. However, if these penalties are known a priori, the methodology used in this paper can be straightforwardly used to consider practical codes and practical implementations of high-order modulation formats. This is left for future investigation.

\section{REFERENCES}

[1] A. Chralyvy, "Plenary paper: The coming capacity crunch," in Proc. European Conf. Optical Communication, Vienna, Austria, Sep. 2009. 
[2] D. J. Richardson, "Filling the light pipe," Science, vol. 330, no. 6002, pp. 327-328, Oct. 2010.

[3] M. Jinno, B. Kozicki, H. Takara, A. Watanabe, Y. Sone, T. Tanaka, and A. Hirano, "Distance-adaptive spectrum resource allocation in spectrumsliced elastic optical path network," IEEE Commun. Mag., vol. 48, no. 8, pp. 138-145, Aug. 2010.

[4] Y. Li, H. Dai, G. Shen, and S. K. Bose, "Adaptive FEC selection for lightpaths in elastic optical networks," in Proc. Optical Fiber Communication Conf., San Francisco, CA, USA, Mar. 2014.

[5] Y. Li, H. Dai, G. Shen, and S. K. Bose, "Adaptive FEC-based lightpath routing and wavelength assignment in WDM optical networks," Opt. Switching Netw., vol. 14, no. 3, pp. 241-249, Aug. 2014.

[6] S. Gringeri, N. Bitar, and T. J. Xia, "Extending software defined network principles to include optical transport," IEEE Commun. Mag., vol. 52, no. 3, pp. 32-40, Mar. 2013.

[7] L. Szczecinski and A. Alvarado, Bit-Interleaved Coded Modulation: Fundamentals, Analysis and Design. Chichester, UK: John Wiley \& Sons, 2015.

[8] H. Bülow, Ü. Abay, A. Schenk, and J. B. Huber, "Coded modulation of polarization- and space-multiplexed signals," in Proc. Asia Communications Photonics Conf. Exhibition, Shanghai, China, Nov. 2011.

[9] D. S. Millar, T. Koike-Akino, R. Maher, D. Lavery, M. Paskov, K. Kojima, K. Parsons, B. C. Thomsen, S. J. Savory, and P. Bayvel, "Experimental demonstration of 24-dimensional extended Golay coded modulation with LDPC," in Proc. Optical Fiber Communication Conf., San Francisco, CA, USA, Mar. 2014.

[10] A. Alvarado and E. Agrell, "Four-dimensional coded modulation with bit-wise decoders for future optical communications," J. Lightw. Technol., vol. 33, no. 10, pp. 1993-2003, May 2015.

[11] A. Alvarado, E. Agrell, D. Lavery, R. Maher, and P. Bayvel, "Replacing the soft-decision FEC limit paradigm in the design of optical communication systems (Invited Paper)," J. Lightw. Technol., vol. 33, no. 20, pp. 43384352, Oct. 2015.

[12] A. Nag, M. Tornatore, and M. Mukherjee, "Optical network design with mixed line rates and multiple modulation formats," J. Lightw. Technol., vol. 28, no. 4, pp. 466-475, Feb. 2010.

[13] G.-H. Gho, L. Klak, and J. M. Kahn, "Rate-adaptive coding for optical fiber transmission systems," J. Lightw. Technol., vol. 29, no. 2, pp. 222 233, Jan. 2011.

[14] G.-H. Gho and J. M. Kahn, "Rate-adaptive modulation and low-density parity-check coding for optical fiber transmission systems," J. Opt. Commun. Netw., vol. 4, no. 10, pp. 760-768, Oct. 2012

[15] D. A. A. Mello, A. N. Barreto, T. C. de Lima, T. F. Portela, L. Beygi, and J. M. Kahn, "Optical networking with variable-code-rate transceivers," J. Lightw. Technol., vol. 32, no. 2, pp. 257-266, Jan. 2014.

[16] S. J. Savory, "Congestion aware routing in nonlinear elastic optical networks," IEEE Photon. Technol. Lett., vol. 26, no. 10, pp. 1057-1060, May 2014.

[17] N. Sambo, G. Meloni, F. Cugini, A. D'Errico, L. Potì, P. Iovanna, and P. Castoldi, "Routing, code, and spectrum assignment (RCSA) in elastic optical networks," in Proc. Optical Fiber Communication Conf, Los Angeles, CA, USA, Mar. 2015.

[18] N. Sambo, G. Meloni, F. Cugini, A. D'Errico, L. Potì, P. Iovanna, and P. Castoldi, "Routing code and spectrum assignment (RCSA) in elastic optical networks," J. Lightw. Technol., vol. 33, no. 24, pp. 5114-5121, Dec. 2015.

[19] N. Sambo, G. Meloni, F. Cugini, A. D’Errico, L. Potì, P. Iovanna, and P. Castoldi, "Routing, code, and spectrum assignment, subcarrier spacing, and filter configuration in elastic optical networks (Invited Paper)," J. Opt. Commun. Netw., vol. 11, no. 7, pp. B93-B100, Nov. 2015.

[20] N. Sambo, G. Meloni, F. Paolucci, F. Cugini, M. Secondini, F. Fresi, L. Potì, and P. Castoldi, "Programmable transponder, code and differentiated filter configuration in elastic optical networks," J. Lightw. Technol., vol. 32, no. 11, pp. 2079-2086, Jun. 2014.

[21] K. Christodoulopoulos, I. Tomkos, and E. Varvarigos, "Elastic bandwidth allocation in flexible OFDM-Based optical networks," J. Lightw. Technol., vol. 29, no. 9, pp. 1354-1366, May 2011.

[22] R. Schmogrow, D. Hillerkuss, M. Dreschmann, M. Huebner, M. Winter, J. Meyer, B. Nebendahl, C. Koos, J. Becker, W. Freude, and J. Leuthold, "Real-time software-defined multiformat transmitter generating 64QAM at 28 GBd," IEEE Photon. Technol. Lett., vol. 22, no. 21, pp. 1601-1603, Nov. 2010

[23] K. Roberts and C. Laperle, "Flexible transceivers," in Proc. Eur. Conf. Optical Communication, Amsterdam, The Netherlands, Sep. 2012.
[24] H. Y. Choi, T. Tsuritani, and I. Morita, "BER-adaptive flexible-format transmitter for elastic optical networks," Opt. Exp., vol. 20, no. 17, pp. 18 652-18 658, Aug. 2012.

[25] B. T. Teipen, M. H. E. an K. Grobe, and J.-P. Elbers, "Adaptive data rates for flexible transceivers in optical networks," J. Netw., vol. 17, no. 5, pp. 776-782, May 2012.

[26] H. Y. Choi, L. Liu, T. Tsuritani, and I. Morita, "Demonstration of BERAdaptive WSON employing flexible transmitter/receiver with an extended openflow-based control plane," IEEE Photon. Technol. Lett., vol. 25, no. 2, pp. 119-121, Jan. 2013

[27] N. Sambo, F. Paolucci, F. Cugini, M. Secondini, L. Potì, G. Berrettini, G. Meloni, F. Fresi, G. Bottari, and P. Castoldi, "Software defined coderate-adaptive terabit/s based on time-frequency packing," in Proc. Optical Fiber Communication Conf., Anaheim, CA, USA, Mar. 2013.

[28] A. Splett, C. Kurtzke, and K. Petermann, "Ultimate transmission capacity of amplified optical fiber communication systems taking into account fiber nonlinearities," in Proc. Eur. Conf. Optical Communication, Montreux, Switzerland, Sep. 1993.

[29] E. Grellier and A. A. Bononi, "Quality parameter for coherent transmissions with Gaussian-distributed nonlinear noise," Opt. Exp., vol. 19, no. 13, pp. 12 781-12 788, Jun. 2011.

[30] P. Poggiolini, A. Carena, V. Curri, G. Bosco, and F. Forghieri, "Analytical modeling of nonlinear propagation in uncompensated optical transmission links," IEEE Photon. Technol. Lett., vol. 23, no. 11, pp. 742-744, Jun. 2011.

[31] L. Beygi, E. Agrell, P. Johannisson, M. Karlsson, and H. Wymeersch, "A discrete-time model for uncompensated single-channel fiber-optical links," IEEE Trans. Commun., vol. 60, no. 11, pp. 3440-3450, Nov. 2012

[32] P. Johannisson and M. Karlsson, "Perturbation analysis of nonlinear propagation in a strongly dispersive optical communication system," J. Lightw. Technol., vol. 31, no. 8, pp. 1273-1282, Apr. 2013.

[33] P. Poggiolini, G. Bosco, A. Carena, V. Curri, Y. Jiand, and F. Forghieri, "The GN-model of fiber non-linear propagation and its applications (Invited Paper)," J. Lightw. Technol., vol. 32, no. 4, pp. 694-721, Oct. 2014.

[34] P. Johannisson and E. Agrell, "Modeling of nonlinear signal distortion in fiber-optic networks," J. Lightw. Technol., vol. 32, no. 23, pp. 4544-4552, Dec. 2014

[35] J. Zhao, H. Wymeersch, and E. Agrell, "Nonlinear impairment aware resource allocation in elastic optical networks," in Proc. Optical Fiber Communication Conf., Los Angeles, CA, USA, Mar. 2015.

[36] L. Yan, E. Agrell, and H. Wymeersch, "Resource allocation in nonlinear flexible-grid fiber-optic networks," in Proc. Optical Fiber Communication Conf., Los Angeles, CA, USA, Mar. 2015.

[37] L. Yan, E. Agrell, and H. Wymeersch, "Link-level resource allocation for flexible-grid nonlinear fiber-optic communication systems," IEEE Photon. Technol. Lett., vol. 27, no. 12, pp. 1250-1253, Jun. 2015.

[38] D. J. Ives, P. Bayvel, and S. J. Savory, "Adapting transmitter power and modulation format to improve optical network performance utilizing the Gaussian noise model of nonlinear impairments," J. Lightw. Technol., vol. 32, no. 21, pp. 4087-4096, Nov. 2014.

[39] D. J. Ives, P. Bayvel, and S. J. Savory, "Physical layer transmitter and routing optimization to maximize the traffic throughput of a nonlinear optical mesh network," in Proc. Int. Conf. Optical Network Design Modeling, Stockholm, Sweden, May. 2014

[40] D. J. Ives, P. Bayvel, and S. J. Savory, "Routing, modulation, spectrum and launch power assignment to maximize the traffic throughput of a nonlinear optical mesh network," Photon. Netw. Commun., vol. 29, no. 3, pp. 244-256, Mar. 2015

[41] D. J. Ives, P. Bayvel, and S. J. Savory, "Assessment of options for utilizing SNR margin to increase network data throughput," in Proc. Optical Fiber Communication Conf., Los Angeles, CA, USA, Mar. 2015.

[42] A. Alvarado, D. J. Ives, S. J. Savory, and P. Bayvel, "On optimal modulation and FEC overhead for future optical networks," in Proc. Optical Fiber Communication Conf., Los Angeles, CA, USA, Mar. 2015.

[43] S. Makovejs, C. Behrens, R.-P. Braun, S. Ten, C. Towery, I. Roudas, K. Koreshkov, T. Nath, and A. Gladisch, "Impact of adaptive-rate transponders and fiber attributes on the achievable capacity," J. Opt. Commun. Netw., vol. 7, no. 3, pp. 172-175, Mar. 2015.

[44] S. Jain, A. Kumar, S. Mandal, J. Ong, L. Poutievski, A. Singh, S. Venkata, J. Wanderer, J. Zhou, M. Zhu, J. Zolla, U. Hölzle, S. Stuart, and A. Vahdat, "B4: Experience with a globally-deployed software defined wan," SIGCOMM Comput. Commun. Rev., vol. 43, no. 4, pp. 3-14, Aug. 2013.

[45] D. A. A. Mello, A. L. N. Souza, J. D. Reis, J. C. M. Diniz, L. H. H. Carvalho, N. G. Gonzalez, J. R. F. Oliveira, D. S. Arantes, and 
M. H. M. Costa, "Parameter selection in optical networks with variablecode-rate transceivers," in Proc. Int. Conf. Optical Network Design Modeling, Pisa, Italy, May. 2015.

[46] T. Fehenberger, A. Alvarado, P. Bayvel, and N. Hanik, "On achievable rates for long-haul fiber-optic communications," Opt. Exp., vol. 23, no. 7, pp. 9183-9191, Apr. 2015.

[47] M. Ivanov, F. Brännström, A. Alvarado, and E. Agrell, "General BER expression for one-dimensional constellations," in Proc. IEEE Global Telecommunications Conf., Anaheim, CA, USA, Dec. 2012.

[48] G. Ungerboeck, "Channel coding with multilevel/phase signals," IEEE Trans. Inf. Theory, vol. 28, no. 1, pp. 55-67, Jan. 1982.

[49] G. D. Forney, Jr, and G. Ungerboeck, "Modulation and coding for linear Gaussian channels (Invited Paper)," IEEE Trans. Inf. Theory, vol. 44, no. 6, pp. 2384-2415, Oct. 1998.

[50] R. Maher, A. Alvarado, D. Lavery, and P. Bayvel, "Multi-channel DBP for reach enhancement of high capacity M-QAM super-channels," in Proc. Signal Processing Photonic Communications, Boston, MA, USA, Jun./Jul. 2015.

[51] R. Maher, A. Alvarado, D. Lavery, and P. Bayvel, "Modulation order and code rate optimisation for digital coherent transceivers using generalised mutual information," in Proc. Eur. Conf. Optical Communication, Valencia, Spain, Sep. 2015.

Alex Alvarado (S'06-M'11-SM'15) was born in Quellón, on the island of Chiloé, Chile. He received the Electronics Engineer degree (Ingeniero Civil Electrónico) and the M.Sc. degree (Magíster en Ciencias de la Ingeniería Electrónica) from Universidad Técnica Federico Santa María, Valparaíso, Chile, in 2003 and 2005, respectively, and the Degree of Licentiate of Engineering (Teknologie Licentiatexamen) and Ph.D. degree, both from Chalmers University of Technology, Gothenburg, Sweden, in 2008 and 2011, respectively.

He is currently a Senior Research Associate at the Optical Networks Group, University College London, London, U.K. During 2012-2014, he was a Marie Curie Intra-European Fellow with the University of Cambridge, Cambridge, U.K., and during 2011-2012 he was a Newton International Fellow with the same institution. In 2008, he was the Holder of the Merit Scholarship Program for Foreign Students, granted by the Ministère de l'Éducation, du Loisir et du Sports du Québec. His general research interests include the areas of digital communications, coding, and information theory. He received the 2009 IEEE Information Theory Workshop Best Poster Award, the 2013 IEEE Communication Theory Workshop Best Poster Award, and the 2015 IEEE TRANSACTION ON COMMUNICATIONS Exemplary Reviewer Award.

David J. Ives received the B.Sc. degree in physics from the University of Birmingham, Birmingham, U.K., in 1988, and the M.Res. degree in photonic systems development and the Ph.D. degree in engineering from University College London (UCL), London, U.K., in 2011 and 2015, respectively.

His interest in optical fiber and optical communications began at the National Physical Laboratory in Teddington, U.K., as a part of the Photonics Group. For more than 21 years, he developed and maintained techniques to characterize the transmission properties of optical fiber and for the calibration of optical fiber test equipment.

In September 2010, he joined the Centre for Doctoral Training in Photonic Systems Development at UCL and in 2011 started the Ph.D. work with the Optical Networks Group. His research interest includes physical layer optical network optimization to improve network resource utilization and maximizing the throughput. In 2015, this led to his Ph.D. dissertation titled "Coherent Optical Fiber Networking in the Nonlinear Regime." He is currently working to create robust abstractions of the physical layer suitable for the creation and control of coexisting virtual networks.
Seb J. Savory (M'07-SM'11) received the M.Eng., M.A., and Ph.D. degrees in engineering from the University of Cambridge, Cambridge, U.K., in 1996, 1999, and 2001, respectively, and the M.Sc. (Maths) degree in mathematics from the Open University, Milton Keynes, U.K., in 2007.

His interest in optical communications began in 1991, when he joined STL (subsequently Nortel) in Harlow, U.K. Having been sponsored by Nortel through his undergraduate and postgraduate studies, he rejoined the Harlow Laboratories in 2000. In 2005, he moved to University College London, London, U.K., where he held a Leverhulme Trust Early Career Fellowship from 2005 to 2007, before being appointed as a Lecturer in 2007, a Reader in 2012, and a Professor in 2015. During 2014-2015, he held a RAEng/Leverhulme Trust Senior Research Fellowship and received the RAEng Colin Campbell Mitchell Award in 2015. In October 2015, he was elected as a Fellow of Churchill College, Cambridge, U.K., and in January 2016, moved to Cambridge as a University Lecturer.

Dr. Savory is the Editor-in-Chief of the IEEE PHOTONICS TeCHNOLOGY LETTERS and serves on the Steering Committee of the Optical Fiber Communication conference having previously served as the General Chair in 2015 and the Program Chair in 2013.

Polina Bayvel received the B.Sc. (Eng.) and Ph.D. degrees in electronic and electrical engineering from the University of London, London, U.K., in 1986 and 1990, respectively. In 1990, she was with the Fiber Optics Laboratory, General Physics Institute, Russian Academy of Sciences, Moscow, Russia, under the Royal Society Postdoctoral Exchange Fellowship. She was a Principal Systems Engineer with STC Submarine Systems, Ltd., London, U.K., and Nortel Networks, Harlow, U.K., and Ottawa, ON, Canada, where she was involved in the design and planning of optical fiber transmission networks. During 1994 2004, she held a Royal Society University Research Fellowship with University College London (UCL), and in 2002, she became a Chair in Optical Communications and Networks. She is the Head of the Optical Networks Group (ONG), UCL, which she also set up in 1994. She has authored or coauthored more than 300 refereed journal and conference papers. Her research interests include wavelength-routed optical networks, high-speed optical transmission, and the study and mitigation of fiber nonlinearities.

She is a Fellow of the Royal Academy of Engineering, the Optical Society of America, the U.K. Institute of Physics, and the Institute of Engineering and Technology. She received the Royal Society Wolfson Research Merit Award (2007-2012), the 2013 IEEE Photonics Society Engineering Achievement Award, and the 2014 Royal Society Clifford Patterson Prize Lecture and Medal. In 2015, she and five members of ONG received the Royal Academy of Engineering Colin Campbell Mitchell Award for their pioneering contributions to optical communications technology. 